\title{
STARK BROADENING PARAMETERS OF C IV LINES FOR STELLAR PLASMA RESEARCH
}

\author{
M.S. DIMITRIJEVIC \\ Astronomical Observatory, Volgina 7, 11050 Beograd, Yugoslavia \\ and \\ S.SAHAL-BRECHOT \\ Observatoire de Paris-Meudon, 92190 Meudon, France
}

In order to complete available C IV broadening data needed for stellar plasma research, we have calculated Stark broadening parameters for $69 \mathrm{C}$ IV multiplets of large principal quantum number. The results along with a discussion of the Stark broadening parameter regularities within spectral series will be published elsewhere (Dimitrijevic and Sahal-Brechot, 1992). As an example in Figs 1 and 2 the case of C IV $n p^{2} P^{0}-9 s^{2} S$ transitions, is presented. We can see that particularly for shifts the changes of Stark broadening parameters are relatively small, permitting the interpolation of new data or critical evaluation of mutual consistency of existing data.

\section{References}

Dimitrijevic, M.S., Sahal-Brechot,S. (1992) $A \& A S$, in press.
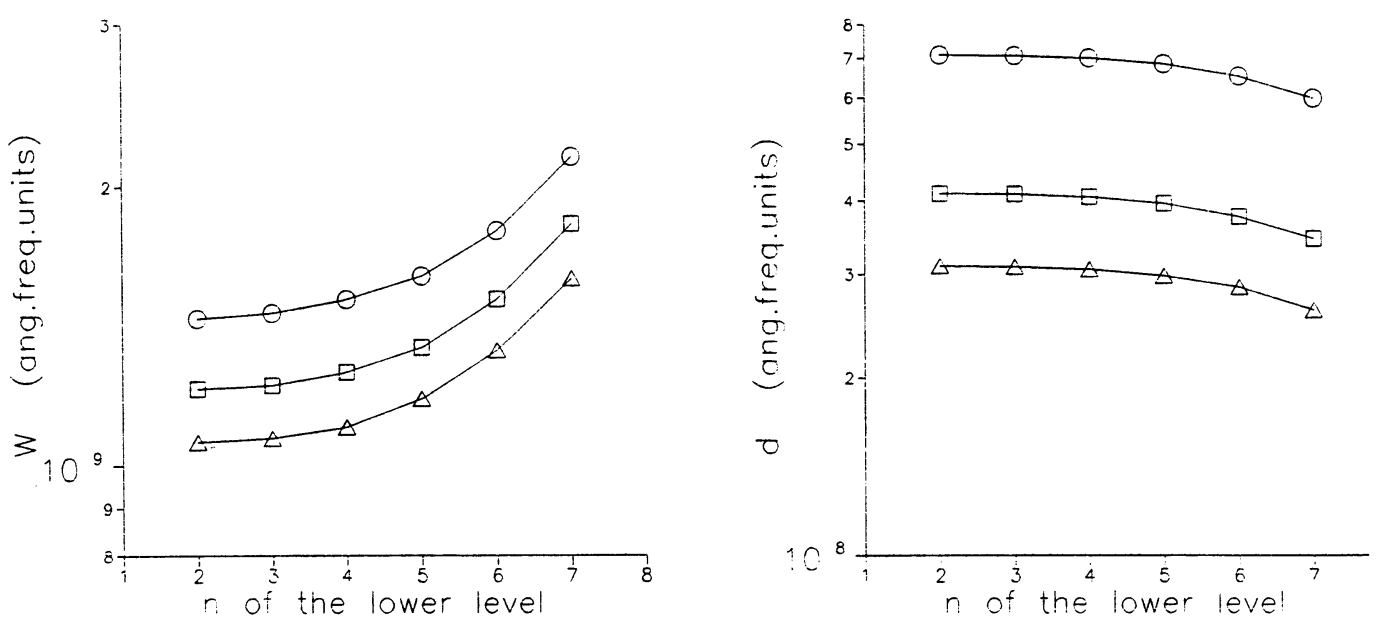

Fig. 1. Figure 1a, 1b. Stark full half widths (1a) and shift (1b) for the C IV $n p^{2} P^{0}-9 s^{2} S$ lines as a function of $n$ for $T=20,000 \mathrm{~K}$ (circles); $T=100,000 \mathrm{~K}$ (squares) and $200,000 \mathrm{~K}$ (triangles) at $N_{e}=10^{13} \mathrm{~cm}^{-3}$ 